\title{
Depression, pain and quality of life in patients with amyotrophic lateral sclerosis: a cross-sectional study
}

\author{
Alessia Pizzimenti, PsyDa,b \\ Massimiliano Aragona, MD \\ Emanuela Onesti, MDa \\ Maurizio Inghilleri, MD, $\mathrm{PhD}^{\mathrm{a}}$
}

a ALS Center, Department of Neurology and
Psychiatry, Sapienza University of Rome, Italy
b Crossing Dialogues Association, Rome, Italy

Correspondence to: Maurizio Inghilleri

E-mail: maurizio.inghilleri@uniroma1.it

\section{Summary}

The complications related to amyotrophic lateral sclerosis (ALS) include pain. A higher risk of depression and a negative effect on quality of life (QoL) might be expected in ALS patients with pain.

The aims of this study were to evaluate the prevalence of pain in ALS patients, to compare measures of depression and QoL in patients with and without pain, and to study the influence of depression scores and pain on the QoL of ALS patients with pain.

Forty ALS patients were enrolled, and 36 were included in the analysis. Seventy-two percent of patients reported pain. Pain intensity was significantly related to a worsening of QoL $(p<.05)$. This effect was no longer significant after considering depression scores as a covariate. Depression scores significantly decreased $Q o L(p<.02)$ and this effect remained significant after considering pain intensity as a covariate (p<.05).

Our study suggests that pain is frequent in ALS patients and that depressive symptoms are significantly related to poorer QoL. Clinicians should pay more attention to both pain and depressive symptoms in ALS patients considering their effect on QoL.

KEY WORDS: amyotrophic lateral sclerosis, depression, pain, quality of life

\section{Introduction}

Amyotrophic lateral sclerosis (ALS) is a neurodegenerative disease characterized by progressive degeneration of motor neurons in the primary motor cortex, brainstem and spinal cord with a heterogeneous clini- cal presentation (Mitchell and Borasio, 2007). The mainstay of ALS management is currently limited to symptomatic treatment and palliative care.

Pain is a possible complication of ALS (Chiò et al., 2012 ); its prevalence ranges from 3 to $78 \%$ and its occurrence is directly proportional to the disease progression (Mitchell and Borasio, 2007; Jensen et al., 2007). As regards the etiology of the pain, the involvement of either peripheral or central mechanisms in ALS is still under debate (Mitchell and Borasio, 2007; Hammad et al., 2007).

Considering the severity of the disease, a high prevalence of depression and a significant decrease in quality of life (QoL) can be expected in patients with ALS. Prevalence rates of depression range from 0 to $44 \%$ and from 9 to $11 \%$ in studies employing structured interviews based on the DSM-IV (Pagnini, 2013). A recent study, using a semi-structured interview and four self-report depression severity measures, reported that the prevalence of major depression in ALS ranges from 16.2 to $25 \%$, depending on the assessment instruments employed (Ferentinos et al., 2011). Moreover, evidence about QoL in ALS patients is conflicting. While some studies suggest a correlation between decreased QoL and disease severity, others report a non-significant correlation between QoL and measures of muscular strength and physical functional status measures (Nelson et al., 2003; Simmons et al., 2000; Chiò et al., 2004; Tramonti et al., 2012). Despite these variable data, there is a general consensus that life satisfaction in ALS patients does not depend only on the level of physical impairment but is, rather, part of a complex reaction to several elements, including the level of social and financial support, psychological and existential factors, and disease duration (Simmons et al., 2000; Nelson et al., 2003; Chiò et al., 2004; Tramonti et al., 2012).

In other medical conditions, a relationship between pain, depression and poor QoL is supported by evidence (Stafford et al., 2007; Van Voorhees and Fried, 2009). Pain is often responsible for secondary depression and vice versa, and some authors propose common pathophysiological mechanisms or cognitive mediators linking these conditions to explain this clinical correlation (Robinson et al., 2009; Radat and Koleck, 2011). Patients with multiple sclerosis (MS) suffer from various painful conditions like those found in ALS, and a close relationship between pain, depression and poor QoL has recently been highlighted in MS (Truini et al., 2013). Consequently, a higher risk of depression with a negative effect on QoL might be expected in ALS patients with pain. Conversely, previ- 
ous research has produced variable results on the direct relationship between depression and QoL in ALS patients (Kübler et al., 2005; Gauthier et al., 2007; Lulé et al., 2008; Tramonti et al., 2012). A significant negative correlation between low depression score and high QoL was found by Lulé et al. (2008). However, this evidence is not very strong and a more complex relationship has been suggested, in which depressive symptoms seem to be related more to health-related QoL than to subjective life satisfaction reports (Tramonti et al., 2012).

With regard to the influence of pain on QoL, the general view is that pain in ALS is a frequent symptom especially in the later stages of the disease and can have a pronounced influence on QoL and suffering. However, in ALS patients, pain and depression are under-recognized and undertreated by physicians, and direct evidence is lacking and conflicting (Pagnini et al., 2012). Further research is needed to better understand the currently debated mechanisms underlying the link between depression and pain in ALS (e.g., primary versus secondary depression, common pathophysiology, etc.).

Studies on pain in ALS could prove very significant given that pain might be related to subjective distress, secondary depression, and reduced mobility, all factors possibly related, in turn, to reduction of QoL (Banks and Kerns, 1996). However, current evidence on the relationship between pain, depression and QoL in ALS patients is limited in quantity and partially conflicting, thus specific studies on the interplay between these dimensions are warranted.

Accordingly, the aims of this study were: i) to evaluate the prevalence of pain in ALS patients; ii) to compare measures of depression and QoL in ALS patients with and without pain; and iii) to study the influence of depression scores and pain on the QoL of ALS patients with pain.

\section{Materials and methods}

Over a six-month period, a group of consecutive patients with a clinical diagnosis of familial or sporadic ALS according to the El Escorial criteria (Brooks et al., 2000) and attending the multidisciplinary outpatients' ALS Center at the Sapienza University of Rome, were enrolled in this study. All the patients underwent clinical evaluation using the Medical Research Council scale for muscle strength (MRC) and the ALS Functional Rating Scale (ALS-FRS) (Medical Research Council, 1976), while QoL was evaluated using the Quality of Life Index (QL-Index) (Spitzer et al., 1981), depression using the Zung Self-Rating Depression Scale (SDS) (Zung et al., 1965), and pain intensity using a visual analog scale (VAS) (McCormack et al., 1988).

The localization of pain was assessed clinically while two items of the Italian version of the Neuropathic Pain Symptom Inventory were used to establish the duration and frequency of pain in the last 24 hours (Padua et al., 2009). These last items were scored on a Likert scale as follows: duration from 0 (less than 1 hour) to 4 (continuous), frequency from 0 (no pain at all) to 4 (more than 20 pain episodes). Patients with a VAS score between 0 and $2 \mathrm{~mm}$ were considered pain-free (Aicher et al., 2012).

We deliberately did not describe the kind of pain presented by patients (neuropathic or non-neuropathic) because our purpose was only to evaluate the relationship of QoL and depression with perceived pain.

The study was approved by the local ethics committee.

\section{Statistics}

Statistical analyses were performed using the Statistical Package for Social Sciences, version 16.0 (SPSS Inc., Chicago, IL, USA). Descriptive statistics were calculated and expressed as mean values \pm standard deviation. Bivariate analyses were stratified by presence/absence of pain. The Mann-Whitney $U$ and $\chi^{2}$ tests were used to analyze differences between ALS patients with and without pain. Linear regression analyses were used to study the influence of pain on depression and QoL, and of depression on QoL, adjusting for significant covariates when appropriate. $\mathrm{P}$-values less than .05 (two-sided) were considered significant.

\section{Results}

Forty patients were initially enrolled in the study. Four were subsequently excluded because they were not tested on all the study variables. The remaining 36 patients were included in the analysis.

Twenty-six patients $(72.2 \%)$ reported pain, localized as follows: scapular-humeral area (15 patients, $57.7 \%)$, lower limb (8 patients, 30.8\%), cervical-dorsal area (3 patients, $11.5 \%)$. Using Aicher's criteria, the patients' pain was categorized as mild (1 patient, 3.8\%), moderate (3 patients, $11.5 \%$ ), severe (5 patients, 19.2\%), very severe (6 patients, $23.1 \%$ ), and the most severe imaginable (11 patients, 42.3\%) (Aicher et al., 2012). Mean pain intensity was $7.6( \pm 2.7)$, while mean pain duration in the last 24 hours was rated as $1.4( \pm 1.6)$, which corresponds to about two hours per day. The frequency of pain episodes in the last 24 hours was 0.6 $( \pm 1)$ (about two per day).

An SDS total score of 50 or above, indicating depression, was found in only one patient. Sixteen patients self-reported several depressive symptoms but remained in the not depressed range (score between 35 and 47). Ten of the patients scoring 35 or above on the SDS were taking antidepressants.

Table I compares the demographic and clinical characteristics of the patients. No significant differences were found between patients with or without pain relative to gender, type of ALS, age at onset, disease duration, MRC total score, ALS-FRS score, QoL-Index and SDS score. Compared with the pain-free ALS patients, the ALS patients with pain tended to report lower QoL and 
higher depression scores, although these differences were not significant $(p=.52$ and $p=.67$, respectively). The duration and the frequency of pain were not significantly related to either depression or QoL scores, and the intensity of the pain did not significantly influence depression scores. On the other hand, both intensity of pain and depression scores were significantly related to a worsening of $\mathrm{Q} o \mathrm{~L}$ ( $\mathrm{p}<.05$ and $\mathrm{p}<.02$, respectively), although the effect of pain intensity was no longer significant after considering depression scores as a covariate (Table II).

\section{Discussion}

This study was designed to improve our understanding of depression and QoL in patients with ALS and pain. It shows that pain is frequent in ALS patients (72\% reported at least one painful symptom). In our sample, pain was scored as quite distressing (more than 7 on a 10-point pain intensity scale). Previous studies have reported a prevalence of pain in ALS ranging from $3 \%$ to $78 \%$ (see introduction) and in our sample the value was at the upper end of this range. One possible reason for this wide variation is that ALS is often described as characterized by "painless, pro- gressive, but asymmetrical muscle weakness" (Eisen, 2001). This could have led to under-recognition of pain in clinical settings, while pain is probably far more common when studied systematically (Wicks, 2012). Alternatively it could be attributable to differences in the stage of the disease at the time of the study. However, recent evidence showing no statistically significant difference in the presence of pain between patients in various stages of ALS suggests that the effect of the disease duration might be rather weak and that the first hypothesis should be preferred (Rivera et al., 2013). Moreover, unexpectedly, our study did not confirm the correlation between pain and either worse functional score or longer disease duration found in previous studies (Chiò et al., 2012; Rivera et al., 2013). However, our results might be biased by the small sample size, and further studies on larger samples are therefore needed.

Although ALS patients with pain tended to score higher on depression and lower on QoL, these differences did not reach significance. Moreover, in regression analyses pain was not significantly related to depression scores. Pain intensity was significantly related to a reduction in QoL, which is consistent with previous studies showing that pain intensity predicts a worsening of QoL in ALS patients (Pagnini et al., 2012).

Table I - Demographic and clinical characteristics of the patients.

\begin{tabular}{llll}
\hline & $\begin{array}{l}\text { All Patients } \\
(\mathrm{n}=36)\end{array}$ & $\begin{array}{l}\text { Patients with pain } \\
(\mathrm{n}=26)\end{array}$ & $\begin{array}{l}\text { Patients without pain } \\
(\mathrm{n}=10)\end{array}$ \\
\hline Sex & $14 \mathrm{~F}, 22 \mathrm{M}$ & $12 \mathrm{~F}, 14 \mathrm{M}$ & $2 \mathrm{~F}, 8 \mathrm{M}$ \\
Age (years), mean \pm SD & $63.7 \pm 10.9$ & $63.5 \pm 10.6$ & $57.8 \pm 12$ \\
Disease duration (months), mean \pm SD & $22 \pm 14$ & $20 \pm 13$ & $25.6 \pm 19.3$ \\
Age at onset, mean \pm SD & $62 \pm 11$ & $64 \pm 11$ & $58 \pm 12$ \\
ALS-FRS score, mean \pm SD & $35.1 \pm 8.7$ & $34.8 \pm 9$ & $35.9 \pm 8.8$ \\
Clinical onset & 28 spinal, 8 bulbar & 21 spinal, 5 bulbar & 7 spinal, 1 bulbar \\
MRC total score, mean $\pm S D$ & $109.3 \pm 32.2$ & $109.3 \pm 32.4$ & $109.5 \pm 33.3$ \\
QoL, mean $\pm S D$ & $6.9 \pm 1.9$ & $6.5 \pm 1.6$ & $7.9 \pm 2.3$ \\
SDS score, mean $\pm S D$ & $35.6 \pm 8.1$ & $37.4 \pm 8.4$ & $31.2 \pm 5.3$ \\
\hline
\end{tabular}

Abbreviations: $\mathrm{F}=$ females; $\mathrm{M}=$ males; $\mathrm{SD}=$ standard deviation; ALS-FRS=Amyotrophic Lateral Sclerosis Functional Rating Scale; MRC=Medical Research Council scale; QoL=Quality of Life Index; Zung SDS=Zung Self-Rating Depression Scale

Table II - Linear regression models of pain and depression scores (independent variables) on quality of life (dependent variable).

\begin{tabular}{lllllll}
\hline Model & Pain intensity & & \multicolumn{3}{c}{ Zung Self-Rating Depression Scale score } \\
\hline & $\mathrm{B}$ & $\mathrm{SE}$ & $\mathrm{p}$ & $\mathrm{B}$ & $\mathrm{SE}$ & $\mathrm{p}$ \\
1 & -.14 & .07 & .048 & - & - & - \\
2 & - & - & - & -.09 & .04 & .014 \\
3 & -.09 & .07 & .145 & -.08 & .04 & .04 \\
\hline
\end{tabular}

Abbreviations: $B=$ beta coefficient; $S E=$ standard error; $p=p$-value

Model 1: Direct effect of pain intensity on the Quality of Life (QoL); Model 2: Direct effect of depression scores on QoL; Model 3: Concurrent effect of pain and depression scores on QoL 
However, in our sample this effect was not very strong $(p=0.48)$ and was no longer significant after depression scores were added as a possible interfering covariate. Considering that depression is possibly part of the pathway from pain to QoL, the confounding effect of depression scores on the influence of pain on QoL is not per se proof that pain is not independently involved in the worsening of patients' QoL.

As in previous studies, the reported depression scores in our sample were usually in the non-depression range. Nonetheless, their effect was significant, our study showing a negative correlation between depression scores and QoL that remained significant after pain was added as a possible covariate. This finding is in line with previous studies (Kubler et al., 2005; Lulé et al., 2008; Tramonti et al., 2012) and suggests that even sub-threshold depressive symptoms might be actively treated due to their negative effect on the QoL of ALS patients.

Finally, the methodological limitations of our study should be considered, namely, the small sample size, the absence of a control group, the possible influence of treatment with antidepressants on reported depression scores, and the questionable reliability, in neurological diseases, of depression assessments performed using self-rating scales designed for psychiatric depression (due to an overlap between some ALS symptoms and somatic complaints in depression, as well as to possible confusion between subjective experiences of demoralization in ALS and truly depressed mood).

In conclusion, our study suggests that pain is frequent in ALS patients and that depressive symptoms, although scoring under the diagnostic threshold, are significantly related to poorer QoL. As a consequence, clinicians should pay more attention to detecting pain and depressive symptoms in ALS patients and treat them appropriately when necessary.

Further studies are needed to reproduce the present findings with additional measures of pain and functional impairment and to test the efficacy of specific treatments for pain and depression on the QoL of ALS patients.

\section{References}

Aicher B, Peil H, Peil B, et al (2012). Pain measurement: Visual Analogue Scale (VAS) and Verbal Rating Scale (VRS) in clinical trials with OTC analgesics in headache. Cephalalgia 32:185-197.

Banks SM, Kerns RD (1996). Explaining high rates of depression in chronic pain: a diathesis-stress framework. Psychol Bull 119: 95-110.

Brooks BR, Miller RG, Swash M, et al (2000). El Escorial revisited: revised criteria for the diagnosis of amyotrophic lateral sclerosis. Amyotroph Lateral Scler Other Motor Neuron Disord 1: 293-299.

Chiò A, Gauthier A, Montuschi A, et al (2004). A cross sectional study on determinants of quality of life in ALS. J Neurol Neurosurg Psychiatry 75: 1597-1601.

Chiò A, Canosa A, Gallo Set al (2012). Pain in amyotrophic lateral sclerosis: a population-based controlled study. Eur $\mathrm{J}$
Neurology 19: 551-555.

Eisen A (2001). Clinical neurophysiology of ALS. Clin Neurophysiol 112:2190-201.

Ferentinos P, Paparrigopoulos T, Rentzos M et al (2011). Prevalence of major depression in ALS: comparison of a semi-structured interview and four self-report measures. Amyotroph Lateral Scler 12: 297-302.

Gauthier A, Vignola A, Calvo A, et al (2007). A longitudinal study on quality of life and depression in ALS patient-caregiver couples. Neurology 68: 923-926.

Hammad M, Silva A, Glass J, et al (2007). Clinical, electrophysiologic and pathologic evidence for sensory abnormalities in ALS. Neurology 69: 2236-2242.

Jensen MP, Chodroff MJ, Dworkin RH (2007). The impact of neuropathic pain on health-related quality of life: review and implications. Neurology 68: 1178-1182.

Kubler A, Winter S, Ludolph AC, et al (2005). Severity of depressive symptoms and quality of life in patients with amyotrophic lateral sclerosis. Neurorehabil Neural Repair 19: 182-193.

Lulé D, Häcker S, Ludolph A, et al (2008). Depression and quality of life in patients with amyotrophic lateral sclerosis. Dtsch Arztebl Int 105: 397-403.

McCormack HM, Horne DJ, Sheather S (1988). Clinical applications of visual analogue scales: a critical review. Psychol Med 18:1007-1019.

Medical Research Council (1976). Aids to examination of the peripheral nervous system. Memorandum no. 45, Her Majesty's Stationery Office, London.

Mitchell JD, Borasio GD (2007). Amyotrophic lateral sclerosis. Lancet 369: 2031-2041.

Nelson ND, Trail M, Van JN, et al (2003). Quality of life in patients with amyotrophic lateral sclerosis: perceptions, coping resources, and illness characteristics. J Palliat Med 6: 417-424.

Padua L, Briani C, Jann S, et al (2009). Validation of the Italian version of the Neuropathic Pain Symptom Inventory in peripheral nervous system diseases. Neurol Sci 30:99106.

Pagnini F, Lunetta C, Banfi P, et al (2012). Pain in amyotrophic lateral sclerosis: a psychological perspective. Neurol Sci 33: 1193-1196.

Pagnini F (2013). Psychological wellbeing and quality of life in amyotrophic lateral sclerosis: A review. Int J Psychol 48:194-205

Radat F, Koleck M (2011). Pain and depression: cognitive and behavioural mediators of a frequent association. Encephale 37: 172-179.

Reich M, Lesur A, Perdrizet-Chevallier C (2008). Depression, quality of life and breast cancer: a review of the literature. Breast Cancer Res Treat 110: 9-17.

Rivera I, Ajroud-Driss S, Casey P, et al (2013) Prevalence and characteristics of pain in early and late stages of ALS. Amyotroph Lateral Scler Frontotemporal Degener doi:10.3109/21678421.2012.751614

Robinson MJ, Edwards SE, lyengar S, et al (2009). Depression and pain. Front Biosci 14: 5031-5051.

Simmons Z, Bremer BA, Robbins RA, et al (2000). Quality of life in ALS depends on factors other than strength and physical function. Neurology 55: 388-392.

Spitzer WO, Dobson AJ, Hall J (1981). Measuring the quality of life of cancer patients:

a concise QL-Index for use by physicians. J Chronic Dis 34: 585-597.

Stafford L, Berk M, Reddy P, et al (2007). Comorbid depression and health-related quality of life in patients with coronary artery disease. J Psychosom Res 62: 401-410. 
Tramonti F, Bongioanni P, Di Bernardo C, et al (2012). Quality of life of patients with amyotrophic lateral sclerosis. Psychol Health Med 7: 621-628.

Truini A, Barbanti P, Pozzilli C, et al (2013). A mechanism-based classification of pain in multiple sclerosis. J Neurol 260:351-367.

Van Voorhees AS, Fried R (2009). Depression and quality of life in psoriasis. Postgrad Med 121: 154-161.

Wicks $P$ (2012). Reassessing received wisdom in ALS - pain is common when studied systematically. Eur $\mathrm{J}$ Neurol 19:531-532.

Zung WW, Richards CB, Short MJ (1965). Self-rating depression scale in an outpatient clinic. Further validation of the SDS. Arch Gen Psychiatry 13:508-515. 